\title{
Turning macrophages on, off and on again
}
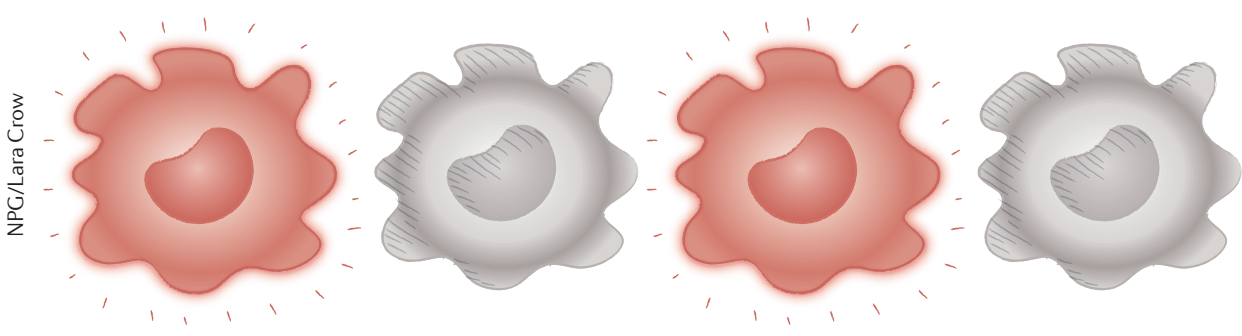

Often, if a tumour responds to therapy, some cells evolve to become resistant and form a secondary tumour. Hemann, Chen and colleagues investigated acquired therapeutic resistance in B cell malignancies.

Pallasch et al. used a humanized mouse model of a chemoresistant $B$ cell malignancy. The malignant $B$ cells initially respond to alemtuzumab (a CD52-specific antibody) and then develop resistance; and so, the authors set about investigating the reasons for this. They implanted resistant malignant B cells into different sites in the humanized mice and found that malignant B cells in the spleen and peripheral blood were sensitive to alemtuzumab, whereas those in the bone marrow developed resistance. Their analyses revealed that alemtuzumab mediates tumour cell death by recruiting macrophages and inducing phagocytosis of tumour cells. An in vivo RNA interference (RNAi) screen revealed that an important mechanism through which therapeutic antibodies can induce tumour cell death inhibitory Fc receptor for IgG (Fc $\gamma$ RIIB) by the malignant B cells in the bone marrow is important for mediating resistance to alemtuzumabinduced macrophage-mediated phagocytosis. Furthermore, prosta- downstream effector of PTGES3, is secreted by alemtuzumab-resistant malignant $B$ cells, which indicates that increased secretion of PGE2 suppresses phagocytosis; the mechanism by which Fc $\gamma$ RIIB mediates resistance to alemtuzumab remains unclear.

Can responses to alemtuzumab be improved? The authors found that alemtuzumab and the alkylating agent cyclophosphamide (but not other alkylating agents) synergistically collaborated to almost entirely eliminate malignant B cells from the bone marrow, and in most cases, a complete response with no relapse after 6 months was achieved in the mice. To understand the mechanism that underlies the synergy of this surprising combination, the authors investigated whether cyclophosphamide affects macrophages in the bone marrow. They found that the combination treatment increased the number of macrophages in the bone marrow and that macrophagemediated phagocytosis was transiently increased. Furthermore, cyclophosphamide treatment caused the transient (24-48 hours) secretion of factors from malignant B cells, including tumour necrosis factor (TNF) and vascular endothelial growth factor A (VEGFA), which were important for the synergy between cyclophosphamide and alemtuzumab. Treatment with cyclophosphamide also reduced the levels of secreted PGE2, which increased macrophage phagocytic activity. This indicates that cyclophosphamide induces a transient secretory response, which the authors call 'acute secretory activation phenotype' (ASAP), in malignant B cells that increase the phagocytic activity of macrophages, which consequently respond to alemtuzumab binding to the malignant $\mathrm{B}$ cells.

The authors further showed that cyclophosphamide synergistically collaborates with other therapeutic antibodies in other mouse models of B cell malignancy. This indicates that the induction of ASAP by cyclophosphamide might be a common mechanism that induces macrophage-mediated phagocytosis of antibody-targeted malignant B cells. Indeed, cyclophosphamide synergistically collaborates with rituximab, which is a CD20-specific antibody that is used to treat various $B$ cell malignancies, in a mouse xenograft model of human B cell acute lymphoblastic leukaemia that exhibited resistance to rituximab monotherapy in the bone marrow.

This study reveals an important mechanism through which therapeutic antibodies can induce tumour cell death, and it gives an insight into how resistance can develop and be overcome. This could have implications for the treatment of patients with B cell malignancies or bone marrow metastases if this synergy also occurs in patients.

Gemma K. Alderton, Senior Editor, Nature Reviews Cancer glandin E2 (PGE2), which is the

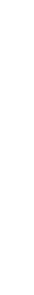

